\title{
Bisphenol A Sensing Device Utilizing Antibody Modified Beads on a Microfluidic Disk
}

\author{
Izumi KUBO, Kazuki Moriwaki, Tomoyuki Kanamatsu, Shunsuke Furutani \\ ${ }^{1}$ Faculty of engineering, Soka University, 1-236 Tangi-cho, Hachioji, Tokyo, 192-8577 JAPAN, \\ Corresponding author's e-mail: kubo@soka.ac.jp
}

\begin{abstract}
Bisphenol A (BPA) is a major material of polycarbonate, which is used as a food container in our life and known as endocrine disruptor. In order to investigate the effect to health animal experiments are necessary. To determine the intake of BPA with food in animals, we need a sensing system of BPA with a small amount of biological sample in short reaction time. We have developed a disk-shaped microfluidic device for ELISA with 32 microchannels and chambers. In order to establish a rapid and sensitive assay system of BPA in biological sample, anti BPA antibody was immobilized on micro beads and introduced into microchambers on the microchannels on the device, in this study. Competitive immunoassay was performed using HRP conjugated BPA with a small amount of sample solution with in $20 \mathrm{~min}$. BPA was determined on the microfluidic disk at the concentration range between 3.9- $250 \mathrm{ng} \mathrm{ml}^{-1}$. BPA spiked rat serum was determined on the disk.
\end{abstract}

Key words: bisphenol A, microfluidic disk, antibody immobilized beads, HRP conjugated bisphenol A, competitive assay, rat serum

\section{Introduction}

Some of artificial chemicals are suspicious about environmental endocrine disruptors. Bisphenol A (BPA) (Fig. 1) is known as one of such chemicals. BPA has been used as a major material to produce polycarbonate, which is widely used in food containers and feeding bottle because of its heat durability and solidity. When hot food or drink is placed into the polycarbonate container, BPA remaining in the plastic tends to dissolve into the food or drink. The eluted BPA from the containers might be consumed with food into the human body. There is a fear on the contamination of BPA in food or drink may have a broad range of adverse effects on public health [1]. In Japan the concentration in food or drink must be lower than $2.5 \mathrm{ppm}$ by food hygiene law. Recently it is susceptible that much lower concentration of BPA may have adverse effect to life. For example, endometriosis or neuronal development at early developmental stage by BPA was reported. Such effect is under investigation by animal experiments. Conventionally, BPA concentration has been measured with the use of HPLC or GC/MS. These apparatuses are expensive and the methods need pretreatment of sample. However, from small animal such as mouse enough amount of sample is not available. Thus, a rapid and easy method for monitoring and analyzing BPA from quite small amount of sample is required. In this study we aimed to establish a BPA sensing system with our original microfluidic ELISA disk-like device.

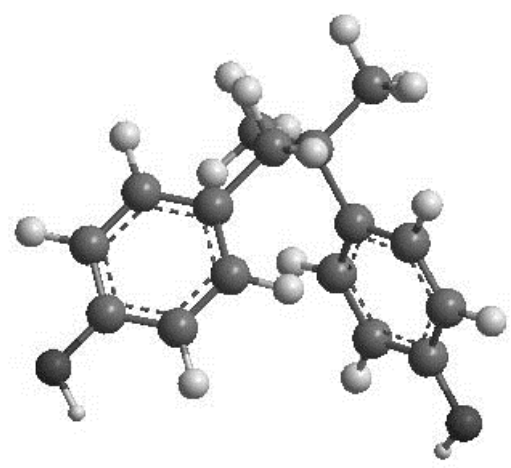

Fig. 1 Structure of BPA 


\section{Materials and Method}

Photoresist SU-8 2035, SU-8 2005, and SU-8 developers were purchased from MicroChem. Polydimethylsiloxane (PDMS), Sylgard 184 silicone elastomer kit, was purchased from World Precision Instruments, Inc. A microfluidic disk $(\phi=10 \mathrm{~cm})$ for the ELISA was designed and microchannels and chambers were fabricated with the use of polydimethyl siloxane (PDMS) [2]. A thick negative photoresist (SU-8 2005) was spun onto a silicon wafer using a spin coater at $3000 \mathrm{rpm}$ for $30 \mathrm{~s}$. Photolithography using a photomask patterned with microchannels was performed according to the usual procedures. When deep microchannels were fabricated, SU-8 2035 was subsequently used at $3000 \mathrm{rpm}$ for $30 \mathrm{~s}$ and exposed through a photomask prior to development. Using the template patterned with SU-8, microchannels were molded out of 1 $\mathrm{mm}$ thick PDMS. As shown in Fig. 2, a PDMS microchannel disk was covered with a glass plate and thirty-two Y-shaped channels were radially designed on one disk. Each channel consisted of two inlets, a detection chamber and an outlet. The channel width was $100 \mu \mathrm{m}$, and the shallow and deep channel depth was 15 and $60 \mu \mathrm{m}$, respectively. Inlets and outlets were made by the perforation of PDMS.

Rabit anti BPA antibody (rabbit anti Bisphenol A-CME-BSA IgG;Cosmobio JAPAN) and HRP conjugated BPA solution (Bisphenol A-4-CPEHRP; Cosmobio JPAN) were used for ELISA of BPA. Rabbit anti BPA antibody was immobilized on the surface of carboxylated beads $(\phi 20 \mu \mathrm{m})$ using water soluble carbodiimide. After blocking, a certain amount of the antibody-immobilized beads were injected into the inlet and introduced into the detection chamber by rotation.

Detection of BPA was performed through competitive immunoassay mixed with HRP conjugated BPA. Schematic illustrations of our bead-based ELISA system are shown in Fig. 3. ELISA steps using the disk were as follows. First, the mixture (one $\mu \mathrm{l}$ ) of equal volumes of sample solution containing BPA and HRP conjugated BPA was injected into inlet (a). The centrifugal disk was rotated to transport the sample solution to the detection chamber to bind to the antibody-immobilized micro beads. Every rotation condition was done at $5000 \mathrm{rpm}$ for $30 \mathrm{sec}$. It was incubated for $15 \mathrm{~min}$ to perform an antigen-antibody reaction. Next, TBST buffer solutions were injected into inlet (a) and the centrifugal disk was rotated to wash the detection chamber. This washing procedure was repeated 5 times to remove unreacted HRP conjugated BPA. Finally, one $\mu l$ of HRP substrate solutions containing luminol was injected into another inlet (b) to avoid the background chemiluminescence (CL) caused by trace amount of HRP conjugated BPA left in the inlet (a). The centrifugal disk was rotated to transport the HRP substrate solution to the detection chamber. Right after the reaction, CL was measured through an imaging analyzer (LAS-3000, FUJIFILM). Every concentration of samples was determined simultaneously with the procedure. ELISA was completed within 20 min with the above procedure in this study, and multiple experiments up to 32 assays were performed with the same disk simultaneously

\section{Results}

Prior to the immobilization of antibody on micro beads, competitive ELISA using a micro titer plate with 96 wells was examined. Rabbit anti BPA was immobilized to the plate. HRP conjugated BPA diluted to $10^{4}$ - fold was mixed with sample solutions and reacted to the antibody for 3 hours. After the reaction, $C L$ of each well was determined. As a result, calibration plot to BPA was obtained and BPA at the range of concentration between 0.97 $1000 \mathrm{ng} / \mathrm{mL}$ was determined.

Then BPA determination using the microfluidic disk was performed. In each determination chamber, $2.0 \times 10^{3}$ of antibody immobilized beads were introduced. Competitive immunoassay was performed by applying the mixture of HRP conjugated BPA diluted to $10^{4}$-fold and BPA solution to the determination chamber and incubated for 15 min. After washing the chamber with $5 \mu$ of buffer solution (TBST), chemi luminescence substrate was introduced into the chamber and detected. As chemiluminescence substrate, luminal and picoluminol (Thermo fisher Scientific) was examined and picoluminol showed enough $\mathrm{CL}$ signal to detect BPA. Dilution rate of BPA conjugated BPA was examined at the rate of $1.0 \times 10^{4}$-fold to $8.0 \mathrm{x}$ $10^{4}$-fold and $2.0 \times 10^{4}$-fold showed the optimum $\mathrm{CL}$ signal. Then under these conditions calibration of BPA was carried out. CL signals depended to the concentration of BPA at the range of $3.9-250 \mathrm{ng} / \mathrm{ml}$ (Fig. 3). This showed that with small amount of sample solution BPA can be determined in much shorter reaction time utilizing antibody immobilized beads on the microfluidic disk. As real serum sample, rat serum spiked by BPA was examined. From the spiked rat serum, BPA was extracted with the use of solid phase extraction column (Isolute SPE M-M; Biotage). The extracted BPA was determined with the microfluidic disk. The spike concentration was 20 and $200 \mathrm{ng} / \mathrm{ml}$ and almost 
consistent $\mathrm{CL}$ signal was obtained on the calibration plot. With the use of the microfluidic disk, volume of sample solution and reaction time was reduced to one tenth compared to micro titer plate assay in this study.

\section{Acknowledgements}

This work was supported in part by a Grant-inAids for Scientific Research (C) from the Ministry of Education, Culture, Sports, Science and Technology (MEXT) Japan and in part by fund from MEXT, the Matching Fund for Private Universities, S1001013, 2010-2015.

\section{References}

[1] Y. Ikezuki, O. Tsutsumi, Y. Takai, Y. Kamei, Y. Taketani, Determination of bisphenol A concentrations in human biological fluids reveals significant early prenatal exposure. Hum. Reprod. 17, 2839-2841(2002);

doi:10.1093/humrep/17.11.2839

[2] N. Matsunaga, S. Furutani, I. Kubo, Centrifugal Flow Device for High-throughput Detection of Cancer Marker CEA, ECS Transactions, 16(11), 123-132 (2008); doi:10.1149/1.2981112
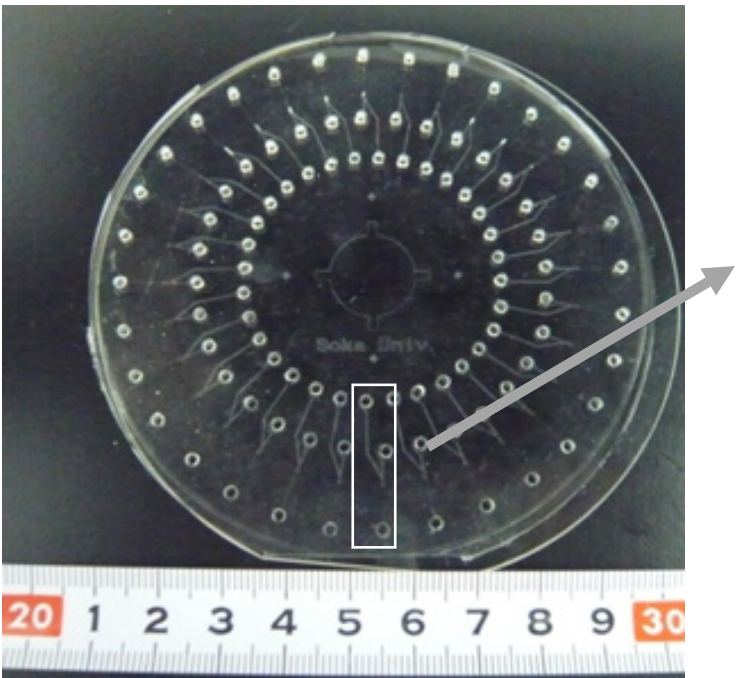

(a) Photograph of a microfluidic disk
Reaction chamber

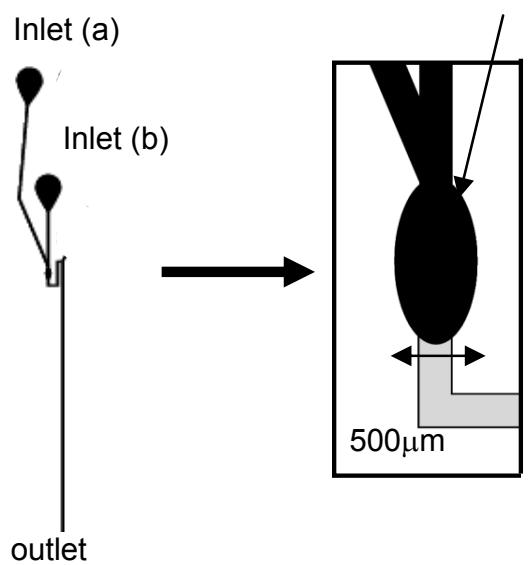

(b) one channel with a reaction chamber

Fig.2 Microfluidic Disk for ELISA

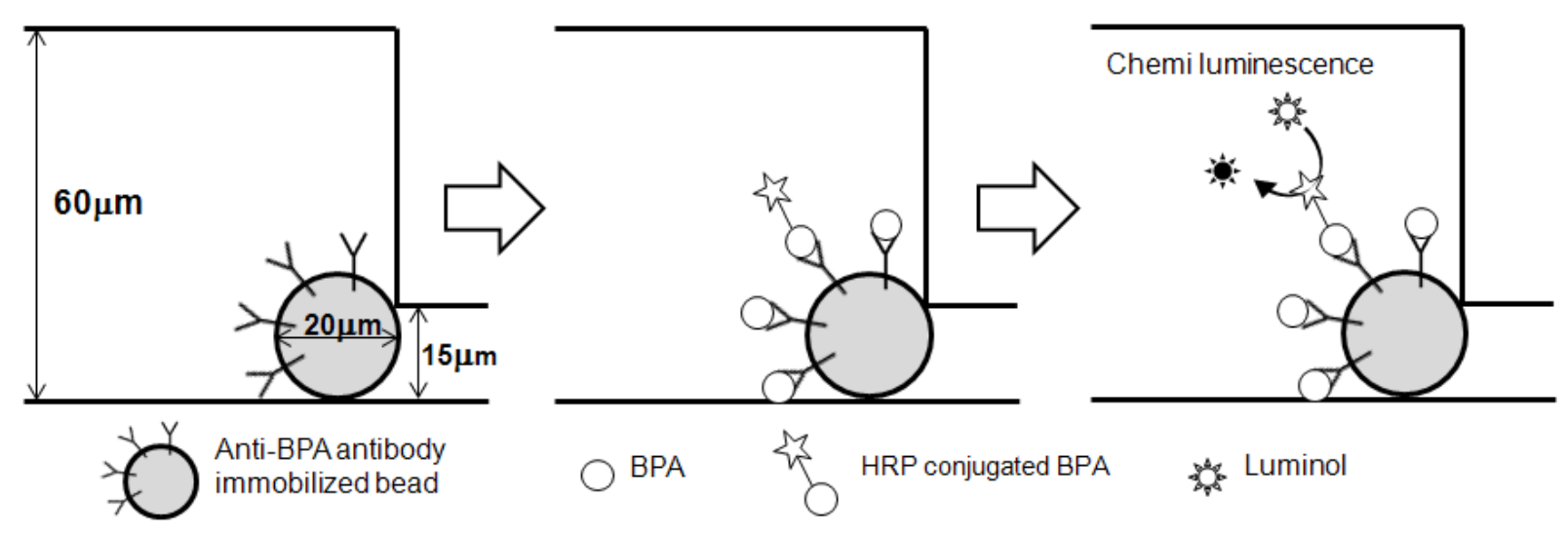

Fig.3 Scheme of ELISA of BPA on a Microfluidic Disk 


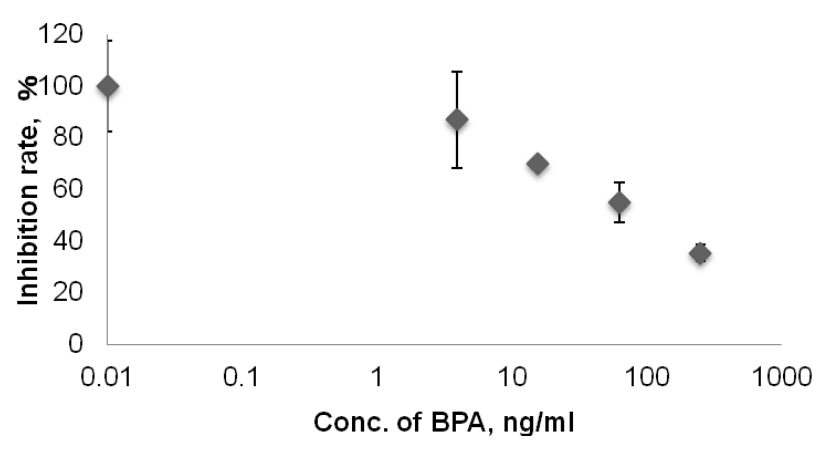

Fig. 4 Calibration plot of BPA by the use of microfluidic disk 